PROCEEDINGS OF THE

AMERICAN MATHEMATICAL SOCIETY

Volume 133, Number 6, Pages 1593-1599

S 0002-9939(04)07693-2

Article electronically published on December 31, 2004

\title{
EXCEPTIONAL CURVES ON SMOOTH RATIONAL SURFACES WITH $-K$ NOT NEF AND OF SELF-INTERSECTION ZERO
}

\author{
MUSTAPHA LAHYANE
}

(Communicated by Michael Stillman)

\begin{abstract}
A $(-n)$-curve is a smooth rational curve of self-intersection $-n$, where $n$ is a positive integer. In 1998 Hirschowitz asked whether a smooth rational surface $X$ defined over the field of complex numbers, having an anticanonical divisor not nef and of self-intersection zero, has $(-2)$-curves. In this paper we prove that for such a surface $X$, the set of $(-1)$-curves on $X$ is finite but non-empty, and that $X$ may have no $(-2)$-curves. Related facts are also considered.
\end{abstract}

\section{INTRODUCTION}

Let $X$ be a smooth rational surface defined over the field of complex numbers such that the self-intersection of its canonical divisor $-K_{X}$ is equal to zero. When $-K_{X}$ is nef (i.e., for every effective divisor $C$ on $X$, the intersection number of $-K_{X}$ with $C$ is greater than or equal to zero), $X$ is a blowing-up of the complex projective plane at nine points, possibly infinitely near.

According to the position of these nine points, in some special cases, many authors were interested in establishing whether the set of $(-1)$-curves on $X$ is finite or not. Thus, Masayoshi Nagata [7] showed that there are infinitely many (-1)curves, provided that the nine points are in general position. Jeffrey Rosoff [ 5 ] treated some cases related to the finite generation of the monoid of effective divisor classes in the Néron-Severi group of the surface. Ulf Persson and Rick Miranda [6] gave a necessary and sufficient condition for the set of $(-1)$-curves on $X$ to be finite when $X$ has a Jacobian elliptic structure. By means of $(-2)$-curves, a complete answer was given in [4], where necessary and sufficient conditions for the set of $(-1)$-curves on $X$ to be finite are given. In particular, it is shown that if the set of $(-1)$-curves on $X$ is finite, then the set of $(-2)$-curves on $X$ is non-empty. In 1998 André Hirschowitz asked whether the latter still holds in the case where $-K_{X}$ is not nef. The aim of this paper is to answer this question in the negative. To this end, we give an explicit construction of a smooth rational surface $X$ defined over

Received by the editors August 27, 2001 and, in revised form, February 23, 2004.

2000 Mathematics Subject Classification. Primary 14J26; Secondary 14F05.

Key words and phrases. Anticanonical rational surfaces, minimal models of smooth rational surfaces, Hodge Index Theorem, points in general position, Néron-Severi group, blowing-up.

This work was partially supported by a postdoctoral fellowship at the International Centre for Theoretical Physics (Trieste, Italy) and by a Marie Curie grant number HPMD-GH-01-00097-01 at the Department of "Álgebra, Geometría y Topología" of the Valladolid University (Valladolid, Spain). 
the field of complex numbers with $-K_{X}$ not nef and no (-2)-curves, and apply the following theorem.

Theorem 1.1. Let $X$ be a smooth rational surface defined over the field of complex numbers such that the following two conditions hold:

1. an anticanonical divisor $-K_{X}$ of $X$ is not nef,

2. $K_{X}^{2}=0$.

Then the set of (-1)-curves on $X$ is finite and non-empty.

The proof of the theorem is postponed to the last section, and we begin with the explicit construction of the desired surface in Section 2.

Surfaces with (-2)-curves are well known to be easily constructed, but it is less obvious whether they contain finitely many $(-1)$-curves. In the third section, we exploit the theorem in this other direction, and use it to construct smooth rational surfaces with finitely many $(-1)$-curves and having some $(-2)$-curves.

Since for any anticanonical rational surface having a Picard number greater than or equal to three, its cone of curves is determined by the reduced irreducible curves of negative self-intersection, the following result holds:

Corollary 1.2. Let $X$ be as in the above theorem. The cone of curves of $X$ is polyhedral.

We fix once and for all a smooth rational surface $X$ over the field of complex numbers, satisfying the two conditions in the theorem. The standard notions, notation and results come from [1], [2] and [3].

\section{A surface With finitely many $(-1)$-Curves and no (-2)-CURVES}

Here we give an example of a smooth rational surface $X$ with $K_{X}^{2}=0$, having a finite number of $(-1)$-curves, and no $(-2)$-curves at all. In fact, in this example, $X$ has no irreducible curve orthogonal to $K_{X}$.

The explicit construction goes as follows: Blow-up a point $P_{1}$ of the projective plane, take the second point $P_{2}$ on the exceptional divisor $E_{1}$ of the blowing-up morphism, then blow-up $P_{2}$ and let $P_{3}$ be the unique intersection point of the strict transform $E_{1}^{2}$ of $E_{1}$ and the exceptional divisor $E_{2}$ of the blowing-up of the point $P_{2}$. The fourth point $P_{4}$ will be the unique point of the intersection of the strict transform $E_{2}^{3}$ of $E_{2}$ and the exceptional divisor $E_{3}$ of the blowing-up of the point $P_{3}$. The fifth point $P_{5}$ is on the intersection of the strict transform $E_{3}^{4}$ of $E_{3}$ and the exceptional divisor $E_{4}$ of the blowing-up of the point $P_{4}$. The sixth point $P_{6}$ is the unique point of the intersection of the exceptional divisor $E_{5}$ of the blowingup of the point $P_{5}$ and the strict transform $E_{4}^{5}$ of $E_{4}$. The seventh point $P_{7}$ is the unique intersection point of the exceptional divisor $E_{6}$ of the blowing-up of the point $P_{6}$ and the strict transform $E_{5}^{6}$ of $E_{5}$. The eighth point $P_{8}$ is the unique point intersection of the exceptional divisor $E_{7}$ of the blowing-up of the point $P_{7}$ and the strict transform $E_{6}^{7}$ of $E_{6}$. Finally, the ninth point $P_{9}$ is the unique intersection point of the strict transform $E_{5}^{8}$ of $E_{5}^{7}\left(=E_{5}^{6}\right)$ with the strict transform $E_{7}^{8}$ of $E_{7}$.

Let $X$ be the surface obtained by blowing-up these nine points. A straightforward calculation shows that $X$ has no irreducible curve orthogonal to a canonical divisor of $X$. In particular, there are no $(-2)$-curves on $X$. To complete the construction, and thus answer Hirschowitz's question in the negative, nothing remains but to prove that $X$ has finitely many $(-1)$-curves. To this end, recall that if $Z$ is the 
projective plane blown-up at $n$ points, then $K_{Z}^{2}=9-n$. Thus, $K_{X}^{2}=0$. Since for example the proper transform $E_{1}^{9}$ of $E_{1}^{3}$ is a (-3)-curve on $X$, it follows that $-K_{X}$ is not nef. Now the theorem applies to show that the set of $(-1)$-curves on $X$ is finite and non-empty.

Remark 2.1. In this example, the cone of curves of $X$ is polyhedral and generated only by the fixed components of $-K_{X}$ and the $(-1)$-curves.

\section{Some EXAmples}

In this section we give some examples of rational surfaces satisfying the conditions of the theorem and having only a finite number of $(-1)$-curves and $(-2)$-curves as well.

Example 3.1. Blow-up a point $P_{1}$ of the projective plane, take the second point $P_{2}$ on the exceptional divisor $E_{1}$ of the blowing-up morphism. Let $P_{3}$ be a general point of the exceptional divisor $E_{2}$ of the blowing-up of the point $P_{2}$, and so on until the ninth point $P_{9}$ which will be the unique intersection point of the exceptional divisor of the blowing-up of the point $P_{8}$ and the strict transform of the exceptional divisor $E_{7}$ associated to the seventh point $P_{7}$. The surface $X$ obtained by blowing up these nine points satisfies the conditions of the theorem; indeed, $-K_{X}$ is not nef since the strict transform of the divisor $E_{7}$ is a $(-3)$-curve, and the other condition $K_{X}^{2}=0$ is obvious. Hence by applying the theorem, $X$ has a finite number of $(-1)$ curves. $X$ has also some $(-2)$-curves, e.g. the strict transform of the exceptional divisor $E_{1}$.

Example 3.2. Consider the surface $X$ obtained by blowing up the projective plane at nine points of a line $L$. Then the strict transform of $L$ is a $(-8)$-curve, and it follows that $-K_{X}$ is not nef. In this special case, one can see that the cone of curves of $X$ is finitely generated by the classes of the exceptional divisors associated to the points in consideration and the strict transform of $L$. One may take more than nine points on a line in the projective plane and obtain a surface such that the self-intersection of its canonical divisor is strictly negative and having a cone of curves finitely generated by the exceptional divisors associated to the points in consideration and the strict transform of the line. In particular, on these surfaces there are no $(-2)$-curves. These examples and many others were constructed by the author after submitting the manuscript containing the example of Section 2

\section{Proof of the theorem}

Recall that $X$ is a smooth rational surface over the field of complex numbers, having a vanishing self-intersection of its canonical divisor.

We will prove that if $X$ has a $(-n)$-curve, $n \geq 3$, then the number of $(-1)$-curves on $X$ is finite. To this end, it is sufficient to consider the following two cases:

(1) $X$ is a blowing-up at 8 points, possibly infinitely near, of $\mathbb{F}_{e}$, where $\mathbb{F}_{e}=$ $\mathbb{P}_{\mathbb{P}^{1}}\left(O_{\mathbb{P}^{1}} \oplus O_{\mathbb{P}^{1}}(e)\right)$ is the Hirzebruch surface associated to the natural integer $e, e \geq 3$

(2) $X$ is a blowing-up at nine points, possibly infinitely near, of the projective plane $\mathbb{P}^{2}$. 
4.1. Case of a blow-up of a Hirzebruch surface. For every divisor $D$ on a smooth projective surface $Y$, let $\bar{D}$ denote the class of $D$ in the Néron-Severi group $N S(Y)$ of $Y$.

Let $\mathbb{F}_{e}$ be the Hirzebruch surface associated to the natural number $e, e \geq 3$. It is well known that $\mathbb{F}_{e}$ has a section $S_{1}$ linearly equivalent to $S_{0}+e F$, where $S_{0}$ (resp. $F$ ) is the section of self-intersection $-e$ (resp. a fibre) of $\mathbb{F}_{e}$ (see e.g. 1], or 3, Theorem 2.17, p. 379]). The group of Néron-Severi of $\mathbb{F}_{e}$ has a basis $\left(\bar{S}_{1}, \bar{F}\right)$ defined by:

- $\bar{S}_{1}$ is the class of the section $S_{1}$ in $\mathrm{NS}\left(\mathbb{F}_{e}\right)$;

- $\bar{F}$ is the class of a fibre $F$ of $\mathbb{F}_{e}$ in $\mathrm{NS}\left(\mathbb{F}_{e}\right)$.

Now let $X$ be the surface obtained by blowing-up at 8 points of the Hirzebruch surface $\mathbb{F}_{e}, e \geq 3$. The group of Néron-Severi $N S(X)$ of $X$ (see e.g. [1], or [3] proposition 3.2 , p. 386$]$ has a basis $\left(\left(\mathcal{S}_{1}, \mathcal{F}\right) ;-\mathcal{E}_{1}, \ldots,-\mathcal{E}_{8}\right)$ defined by:

- $\mathcal{S}_{1}$ is the class of the total transform of the section $S_{1}$ in $X$;

- $\mathcal{F}$ is the class of the total transform of $F$ in $X$;

- $\mathcal{E}_{i}$ is the class of the exceptional divisor corresponding to the $i^{t h}$ blow-up point, $i=1, \ldots, 8$.

The intersection form on $N S(X)$ (see [3, proposition 3.2, p. 386]) is determined by:

- $\mathcal{S}_{1}^{2}=e$

- $\mathcal{F}^{2}=0$

- $\mathcal{E}_{i}^{2}=-1, i=1, \ldots, 8$;

- $\mathcal{S}_{1} \cdot \mathcal{F}=1$

- $\quad \mathcal{S}_{1} \cdot \mathcal{E}_{i}=\mathcal{F} \cdot \mathcal{E}_{i}=0$, for all $i=1, \ldots, 8$

- $\mathcal{E}_{i} \cdot \mathcal{E}_{j}=0$, for every $i, j, i \neq j, i, j \in\{1, \ldots, 8\}$.

If $D$ is a divisor on $X$, then $\bar{D}$ is nef if and only if $D \cdot D^{\prime} \geq 0$ for every effective divisor $D^{\prime}$ on $X$. It is easy to see that:

Lemma 4.1. Let $X$ be the surface obtained by a blowing up at 8 points, possibly infinitely near, of the $\mathbb{F}_{e}$, e a natural integer, $e \geq 3$. Let $S_{1}$ be a section linearly equivalent to $S_{0}+e F$, where $S_{0}$ (resp. $F$ ) is the minimal section (resp. a fibre) of $\mathbb{F}_{e} . S_{0}$ is the section of self-intersection $-e$. Then the class $\mathcal{S}_{1}$ of the total transform of $S_{1}$ (resp. of $F$ ) is nef on $X$.

Our result is:

Proposition 4.2. Let $X$ be a smooth rational surface with $K_{X}^{2}=0$. If $X$ is a blowing-up of $\mathbb{F}_{e}, e \geq 3$, then the number of (-1)-curves on $X$ is finite.

Proof. Let $E$ be a $(-1)$-curve on $X$. Then the group of Néron-Severi $N S(X)$ of $X$ has a basis $\left(\left(\mathcal{S}_{1}, \mathcal{F}\right) ;-\mathcal{E}_{1}, \ldots,-\mathcal{E}_{8}\right)$ (see the notation above). With respect to this basis, the class $\bar{E}$ of $E$ in $N S(X)$ is given by the 10 -tuplet $\left(\left(d, d^{\prime}\right) ; \alpha_{1}, \ldots, \alpha_{8}\right)$ (i.e., $\left.\bar{E}=d \mathcal{S}_{1}+d^{\prime} \mathcal{F}-\alpha_{1} \mathcal{E}_{1}-\ldots-\alpha_{8} \mathcal{E}_{8}\right)$. We have $d^{\prime} \geq 0\left(e \geq 3, \bar{E} \cdot \mathcal{S}_{0} \geq 0\right.$; $\mathcal{S}_{0} \cdot \mathcal{S}_{1}=\mathcal{S}_{0} \cdot \mathcal{E}_{1}=\ldots=\mathcal{S}_{0} \cdot \mathcal{E}_{8}=0$ and $\mathcal{S}_{0} . \mathcal{F}=1$, where $\mathcal{S}_{0}$ is the class of the total transform of $\left.S_{0}\right)$. The same thing holds for $d \geq 0\left(\mathcal{F}\right.$ is nef). Since $\bar{K}_{X}=$ $((-2, e-2) ;-1, \ldots,-1)$, the following equalities hold:

$$
\sum_{i=1}^{i=8} \alpha_{i}^{2}=1+e d^{2}+2 d d^{\prime} ; \sum_{i=1}^{i=8} \alpha_{i}=-1+(e+2) d+2 d^{\prime} .
$$


Now set

$$
\alpha_{i}^{\prime}=\alpha_{i}-\frac{(e+2) d+2 d^{\prime}-1}{8}, i=1, \ldots, 8,
$$

and deduce by the preceding that:

$$
\sum_{i=1}^{i=8} \alpha_{i}^{\prime 2}=-\frac{(e-2)^{2}}{8} d^{2}-\frac{1}{2} d^{\prime 2}-\frac{(e-2)}{2} d d^{\prime}+\frac{1}{4}(e+2) d+\frac{1}{2} d^{\prime}+\frac{7}{8} .
$$

Consequently,

$$
(e-2)^{2} d^{2}+4 d^{\prime 2}+4(e-2) d d^{\prime}-2(e+2) d-4 d^{\prime} \leq 7,
$$

or, equivalently,

$$
((e-2) d)^{2}+(2 d)^{\prime 2}+1+2\left((e-2) d 2 d^{\prime}\right)-2((e-2) d)-2\left(2 d^{\prime}\right) \leq 8 d+8,
$$

which yields

$$
\left((e-2) d+2 d^{\prime}-1\right)^{2} \leq 8 d+8 .
$$

Hence $(d-1)^{2} \leq 8 d+8$, which implies that $d \leq 10$, and since

$$
d^{\prime} \leq \frac{1+\sqrt{8+8 d}}{2}
$$

it follows that $d^{\prime} \leq 5$.

4.2. Case of a blowing-up of the projective plane. Let $X$ be a surface obtained by a blowing-up at 9 points, possibly infinitely near, of the complex projective plane $\mathbb{P}^{2}$. The Néron-Severi group $N S(X)$ of $X$ has a basis $\left(\mathcal{E}_{0},-\mathcal{E}_{1}, \ldots,-\mathcal{E}_{9}\right)$ defined by:

- $\mathcal{E}_{0}$ is the class of a line of $\mathbb{P}^{2}$,

- $\mathcal{E}_{i}$ is the class of the exceptional divisor corresponding to the $i^{t h}$ point blown-up, $i=1, \ldots, 9$.

The intersection form on $N S(X)$ is given by:

- $\mathcal{E}_{0}^{2}=1$

- $\mathcal{E}_{i}^{2}=-1, i=1, \ldots, 9$

- $\mathcal{E}_{i} \cdot \mathcal{E}_{j}=0$, for every $i, j=0,1, \ldots, 9$ with $i \neq j$.

With this in mind, we now proceed to prove the following proposition.

Proposition 4.3. Let $X$ be a smooth rational surface with $K_{X}^{2}=0$. If $X$ is a blowing-up of the projective plane $\mathbb{P}^{2}$ and if $X$ has a $(-n)$-curve $(n \geq 3)$, then the number of $(-1)$-curves on $X$ is finite.

Proof. Let $C$ be a $(-n)$-curve on $X$. With respect to the basis $\left(\mathcal{E}_{0},-\mathcal{E}_{1}, \ldots,-\mathcal{E}_{9}\right)$, the class $\bar{C}$ of the curve $C$ in $N S(X)$ is given by the 10 -tuplet $\left(\delta, m_{1}, \ldots, m_{9}\right)$. Since $C^{2}=-n, C . K_{X}=n-2$ and $\bar{K}_{X}=(-3,-1, \ldots,-1)$, we have

$$
\sum_{i=1}^{i=9} m_{i}^{2}=\delta^{2}+n ; \sum_{i=1}^{9} m_{i}=3 \delta+n-2 .
$$

Let

$$
m_{i}^{\prime}=m_{i}-\frac{3 \delta+n-2}{9}, i=1, \ldots, 9,
$$

so that

$$
\sum_{1}^{9} m_{i}^{\prime 2}=n-\frac{6(n-2) \delta}{9}-\frac{(n-2)^{2}}{9}
$$


by the preceding. Let $E$ be a $(-1)$-curve on $X$. If $\bar{E}=\left(d, \alpha_{1}, \ldots, \alpha_{9}\right)$ is the class of the (-1)-curve $E$ in $N S(X)$ relative to the basis $\left(\mathcal{E}_{0},-\mathcal{E}_{1}, \ldots,-\mathcal{E}_{9}\right)$, then

$$
\sum_{i=1}^{i=9} \alpha_{i}^{2}=d^{2}+1 ; \sum_{i=1}^{9} \alpha_{i}=3 d-1
$$

Write

$$
\alpha_{i}^{\prime}=\alpha_{i}-\frac{3 d-1}{9}, i=1, \ldots, 9
$$

to obtain

$$
\sum_{1}^{9} \alpha_{i}^{\prime 2}=\frac{(6 d+8)}{9}
$$

From the inequality $0 \leq E . C$, we deduce

$$
\sum_{i=1}^{i=9} m_{i}^{\prime} \alpha_{i}^{\prime} \leq \frac{(n-2)}{9}+\frac{\delta}{3}-\frac{(n-2) d}{3} .
$$

But

$$
\left|m_{i}^{\prime}\right| \leq \sqrt{n-\frac{6(n-2) \delta}{9}-\frac{(n-2)^{2}}{9}} ;\left|\alpha_{i}^{\prime}\right| \leq \sqrt{\frac{6 d+8}{9}}, i=1, \ldots, 9,
$$

whence

$$
-\sqrt{(6 d+8)} \sqrt{\left(9 n-6(n-2) \delta-(n-2)^{2}\right.} \leq \sum_{i=1}^{i=9} m_{i}^{\prime} \alpha_{i}^{\prime} \leq \frac{(n-2)}{9}+\frac{\delta}{3}-\frac{(n-2) d}{3} .
$$

Finally,

$$
(3(n-2) d-3 \delta-n+2)^{2} \leq 162(3 d+4)\left(9 n-6(n-2) \delta+(n-2)^{2}\right)
$$

valid for large enough $d$ (i.e., $\left.\frac{(n-2)}{9}+\frac{\delta}{3}-\frac{(n-2) d}{3}<0\right)$ implies that the integer $d$ is bounded.

To end the proof of the theorem, the set of $(-1)$-curves on $X$ is not empty since $X$ is smooth, rational and $K_{X}^{2}=0$.

\section{ACKNOWLEDGEMENTS}

Many thanks to Professors Antonio Campillo, Brian Harbourne, André Hirchowitz, Mudumbai Seshachalu Narasimhan, and Xiao Gang for useful discussions and encouragement. Many thanks also to the referee for the many suggested improvements. This work was done at Laboratoire J.-A. Dieudonné, U.M.R. C.N.R.S. No. 6621, Université de Nice Sophia-Antipolis (Nice, France) and in the Abdus Salam International Centre for Theoretical Physics (Trieste, Italy). The revised enlarged version was done at the Department of Álgebra, Geometría y Topología of the Valladolid University (Valladolid, Spain). I would like to acknowledge the hospitality of these institutions. 


\section{REFERENCES}

1. W. Barth, C. Peters, A. Van de Ven. Compact Complex Surfaces. Berlin, Springer (1984). MR0749574 (86c:32026)

2. B. Harbourne, Anticanonical rational surfaces, Transactions of the American Mathematical Society, Volume 349 (1997), Number 3, 1191-1208. MR1373636 (97f:14007)

3. R. Hartshorne, Algebraic Geometry, Graduate Texts in Mathematics, Springer Verlag (1977). MR0463157 (57:3116)

4. M. Lahyane, Rational Surfaces Having Only a Finite Number of Exceptional Curves. Preprint of The Abdus Salam International Centre for Theoretical Physics, October 2001. Mathematische Zeitschrift, volume 247 (2004), 213-221. MR2054527

5. J. Rosoff, Effective divisor classes and blowings-up of $\mathbb{P}^{2}$. Pacific Journal of Mathematics Vol. 89, No.2, 419-429, 1980. MR0599129 (82e:14042)

6. R. Miranda, U. Persson, On Extremal Rational Elliptic Surfaces. Mathematische Zeitschrift 193, 537-558 (1986). MR0867347 (88a:14044)

7. M. Nagata, On rational surfaces, II, Mem. Coll. Sci. Univ. Kyoto, Ser.E A Math. 33 (1960), 271-293. MR0126444 (23:A3740)

Abdus Salam International Centre for Theoretical Physics, 34100 Trieste, Italy

Current address: Departamento de Álgebra, Geometría y Topología, Facultad de Ciencias, Vallodolid University, 47005 Valladolid, Spain

E-mail address: lahyane@agt.uva.es 